\title{
Technical Notes \\ Experimental Investigation of Hypervelocity Flow in an Expansion Tunnel
}

\author{
C. K. Yuan* \\ State Key Laboratory of High Temperature Gas Dynamic, \\ Chinese Academy of Sciences, 100190 Beijing, \\ People's Republic of China \\ K. Zhou \\ China Academy of Aerospace Aerodynamics, 100074 Beijing, \\ People's Republic of China \\ Z. M. $\mathrm{Hu}^{ \pm}$ \\ State Key Laboratory of High Temperature Gas Dynamic, \\ 100190 Beijing, People's Republic of China \\ and \\ Y. F. Liu尽 and Z. L. Jiang \\ State Key Laboratory of High Temperature Gas Dynamic, \\ Chinese Academy of Sciences, 100190 Beijing, \\ People's Republic of China \\ https://doi.org/10.2514/1.J059086
}

\author{
Nomenclature \\ $L=$ distance between hinge point of double-wedge model and \\ shock-shock interaction point, $\mathrm{mm}$ \\ $R=$ nose radius of the test model, $\mathrm{mm}$ \\ $T=$ translational-rotational temperature, $\mathrm{K}$ \\ $T_{v}=$ vibrational temperature, $\mathrm{K}$ \\ $\Delta=$ shock standoff distance, $\mathrm{mm}$ \\ $\theta=$ polar angle measured from the stagnation line, deg \\ $\varphi=$ polar angle measured from the symmetric axis of the \\ double-wedge model, deg
}

\section{Introduction}

$\mathbf{H}$ YPERVELOCITY flow refers to velocities in the range from a few kilometers per second to some tens of kilometers per second. Different from hypersonic flow, it is characterized by extremely high velocity, and not just a high Mach number. Hypervelocity flow is always accompanied by strong thermochemical

Received 23 September 2019; revision received 14 January 2020; accepted for publication 17 January 2020; published online 24 February 2020. Copyright $(\mathcal{C} 2020$ by the American Institute of Aeronautics and Astronautics, Inc. All rights reserved. All requests for copying and permission to reprint should be submitted to CCC at www.copyright.com; employ the eISSN 1533-385X to initiate your request. See also AIAA Rights and Permissions www.aiaa.org/ randp.

*Senior Engineer, Institute of Mechanics, No. 15 Beisihuanxi Road.

${ }^{\dagger}$ Engineer, No. 15 Yungangxi Road; zkxzh@mail.ustc.edu.cn (Corresponding Author).

${ }^{\ddagger}$ Associate Professor, Institute of Mechanics, No. 15 Beisihuanxi Road; University of Chinese Academy of Sciences, 100049 Beijing, People's Republic of China; huzm@imech.ac.cn.

${ }^{\S}$ Associate Professor, Institute of Mechanics, No. 15 Beisihuanxi Road; University of Chinese Academy of Sciences, 100049 Beijing, People's Republic of China.

"Professor, Institute of Mechanics, No. 15 Beisihuanxi Road; University of Chinese Academy of Sciences, 100049 Beijing, People's Republic of China. nonequilibrium effects and high-temperature gas effects. In addition, hypervelocity flow is a stage that reentry vehicles and planetary probes must go through. To design such vehicles, a computational fluid dynamics (CFD) technique plays an important role in deepening the physical understanding of parametric effects, extrapolating the experimental results to flow situations that are not available in wind tunnels and reducing the design time and cost. Before being used in design work, the technique must be validated against experimental or flight data [1]. Accurate CFD simulations of hypervelocity flow depend on a good knowledge of the chemical species present in the hypervelocity flow, the thermochemical state, chemical processes and reaction rates, energy levels of the radiating species, and the spectral range and distribution of the radiation [2, 3 ]. The shock standoff distance is one of the most common parameters to validate CFD codes. In the past few decades, plenty of experimental $[4,5]$ and theoretical [6-8] studies were conducted to investigate the shock standoff distance. However, the experimental data in hypervelocity flow are sparse [9]. Classic shock standoff distance data are the Lobb sphere data, reporting the shock standoff distance for flow speeds from 2.4 to $6.5 \mathrm{~km} / \mathrm{s}$ over a sphere with a diameter of $0.5 \mathrm{in}$. In 2014 , Zander et al. carried out experiments with diameters of 40,60, and $80 \mathrm{~mm}$ at test flows of 8.7 and $9.7 \mathrm{~km} / \mathrm{s}$ to extend the data [9]. Experiments were also carried out in Large Energy National Shock Tunnels facilities. Due to their relatively long test times, their results mainly concentrated on heat flux and surface pressure measurements [10-12]. To date, knowledge of the hypervelocity flow is far from complete, and more validation data are needed to improve the accuracy of hypervelocity flow models implemented in CFD codes $[\underline{9}, \underline{13}-16]$.

The objective of this work is to conduct experiments with typical models in hypervelocity flow. The results will contribute to deepening the understanding of the physics behind hypervelocity flow processes and to extending the database for CFD validations. Toward this goal, six test models [semisphere, semicylinder, double-cone, double-wedge with different wedge angles, and the simplified Mars Science Laboratory (MSL) entry vehicle models] were selected for the experiments. Details of the shock wave locations, shock standoff distance, shock-shock interaction characteristics, and radiation distribution are presented. In addition, preliminary numerical calculations were performed to try to explain radiation features.

\section{Test Facilities and Flow Conditions}

\section{A. Expansion Tunnel}

Experiments were performed in the JF-16 detonation-driven expansion tunnel, which is capable of achieving freestream conditions with velocities between 5.0 to $10.2 \mathrm{~km} / \mathrm{s}$. The expansion tube also has the benefit of using a variety of test gases, such as air on Earth or gases present in the atmospheres of other planets. The facility consists of six main parts (Fig. 1): the forward detonation cavity detonation tube, the shock tube, the acceleration tube, the nozzle, the test section, and the vacuum section [17]. The facility is driven by a hydrogen/oxygen mixture filled in the detonation tube. The molar ratio of the mixture is determined by the requirements of the experiment.

The expansion tunnel is operated as follows. An igniter is installed at the left end of the detonation tube to initiate forward-running detonation directly. The scored steel primary diaphragm between the detonation and the shock tube bursts after the arrival of the detonation wave; at which time, a shock wave is launched into the shock tube containing the test gas at low pressure. The high-pressure shock accelerates through the second Mylar diaphragm into the acceleration tube, where the test gas is further accelerated by an unsteady expansion wave. The nozzle with an area ratio of 16 


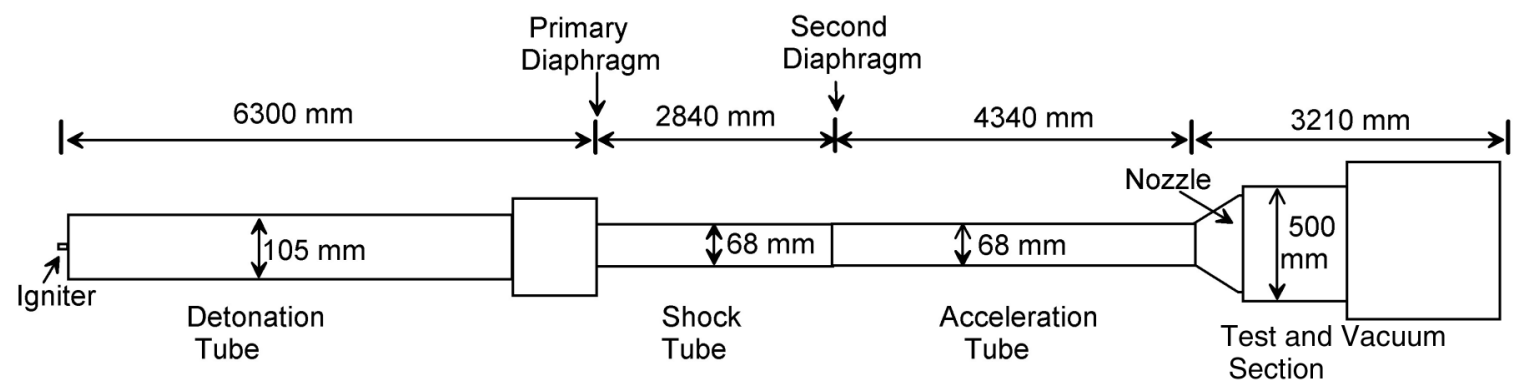

Fig. 1 Schematic of the detonation-driven expansion tunnel: JF-16.

Table 1 Calculated flowfield conditions for the current experiments

\begin{tabular}{lc}
\hline \multicolumn{2}{c}{ current experiments } \\
\hline \hline Parameter & Value \\
\hline Velocity, $\mathrm{m} / \mathrm{s}$ & 7907 \\
Pressure, $\mathrm{Pa}$ & 195 \\
Temperature, $\mathrm{K}$ & 956 \\
Density, $\mathrm{kg} / \mathrm{m}^{3}$ & 0.0006 \\
Mach number & 12 \\
Species mole fractions & \\
Nitrogen $\left(\mathrm{N}_{2}\right)$ & 0.68199 \\
Oxygen $\left(\mathrm{O}_{2}\right)$ & 0.05156 \\
Nitric oxide $(\mathrm{NO})$ & 0.02562 \\
Oxygen atom $(\mathrm{O})$ & 0.24083 \\
Nitrogen atom $(\mathrm{N})$ & 0.0 \\
\hline \hline
\end{tabular}

enlarges the core flowfield at the nozzle exit to approximately $174 \mathrm{~mm}[18]$.

\section{B. Flow Conditions}

Because the effective test time is approximately tens of microseconds, it is difficult to measure the test flow conditions. Numerical methods are needed to calculate the flow parameters by simulating the operation process of the expansion tunnel.

The detonation tube is filled with hydrogen and oxygen at a pressure of 1.0 MPa, whereas the shock tube and the acceleration tube are vacuumized to 3000 and $20 \mathrm{~Pa}$, respectively. Piezoelectric sensors are flush mounted along the shock tube and the acceleration tube to record the static pressure histories and shock arrival time. The shock speed is then obtained using the time-of-flight method. Knowing the initial fill pressure and the measured shock speed, parameters behind the primary shock wave in the shock tube can be obtained by solving shock wave relations. These parameters were further used as initial numerical condition to determine test flow parameters by assuming thermal equilibrium air. More details of the numerical method can be found in Ref. [19]. For the present study, the test gas used is air. Table 1 lists the calculated flowfield conditions.

\section{Instrumentation}

The measurement is based on the assumption that the radiation layer observed around the model approximately equals the shock layer $[9,20]$. In the experiments, a SA4 high-speed color camera takes images from the flow in the test section. At an imaging frame rate of 30,000 frames per second ( $33 \mu$ s interframe time), each image has a charge-coupled device resolution of $320 \times 260$ pixels. The exposure time is set to be $1 \mu \mathrm{s}$. The spatial dimensions are calibrated by imaging a ruled grid placed in the object plane.

\section{Test Model}

To validate the CFD solutions, reliable data of simple geometries are more valuable. Previous studies showed that the blunt-body, double-cone, and double-wedge configurations are sensitive to thermochemical processes in the flow $[5,21]$. The six test models (semisphere, semicylinder, double-cone, double wedge with different wedge angles, and simplified MSL entry vehicle models) were used in this set of experiments. Detailed model dimensions are given in Fig. 2. The span width of the semicylinder and double-wedge models is $50 \mathrm{~mm}$. The test model was positioned in the test section, $10 \mathrm{~mm}$ downstream of the nozzle exit. The nominal angle of attack is $0 \mathrm{deg}$.

\section{E. Image Processing Method}

As the primary goal of this work is to provide data for numerical verification, an image processing algorithm is implemented to extract the shock locations from the images obtained by the high-speed camera. The algorithm assumes that the maximum of the intensity gradient corresponds to the shock location because the flow behind the shock is highly luminous. The processing steps are as follows:

1) Extract a row of the image.

2) Calculate the first derivative of the grayscale.

3) Find the location of the maximum derivative.

4) Repeat these steps for each row of the image.

Figure $\underline{3}$ shows a typical processing result. The uncertainty of the shock wave location obtained by this method depends on the spatial resolution. In the experiments, the uncertainties are $\pm 0.28 \mathrm{~mm}, \pm 0.29 \mathrm{~mm}, \pm 0.285 \mathrm{~mm}, \pm 0.315 \mathrm{~mm}, \pm 0.28 \mathrm{~mm}$, and $\pm 0.285 \mathrm{~mm}$ for the semisphere, semicylinder, MSL, doublecone, 15-35 deg/45 deg double-wedge, and 15-45 deg/55 deg double-wedge models, respectively.

Because the luminosity of hypervelocity flow is intense, the determination of the location of the model is more complicated. The stagnation region of the model is often obscured by the threedimensional nature of the flow [9]. To overcome this problem, an image of the test model illuminated using a planar light source was acquired before each experiment. The aforementioned algorithm was modified to find the minimum of the intensity gradient to profile the

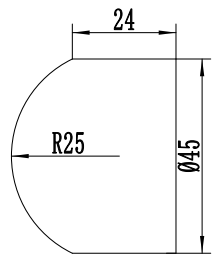

a)

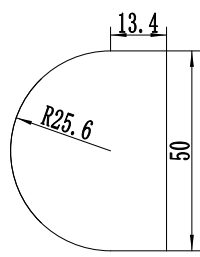

b)

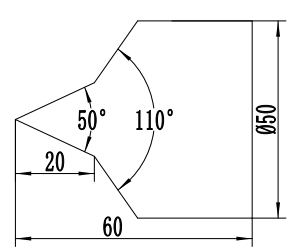

c)

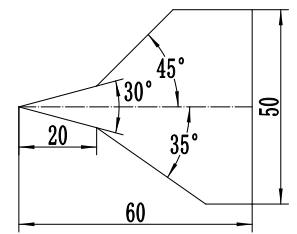

d)

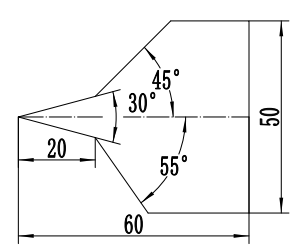

e)

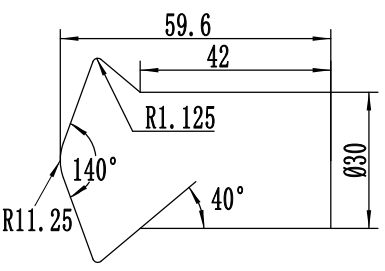

f)

Fig. 2 Test models: a) semisphere, b) semicylinder, c) double-cone, d) double-wedge with second wedge angles of 35 and 45 deg, e) double-wedge with second wedge angle of 45 and $55 \mathrm{deg}$, and f) simplified MSL entry vehicle models. All dimensions are in millimeters. 


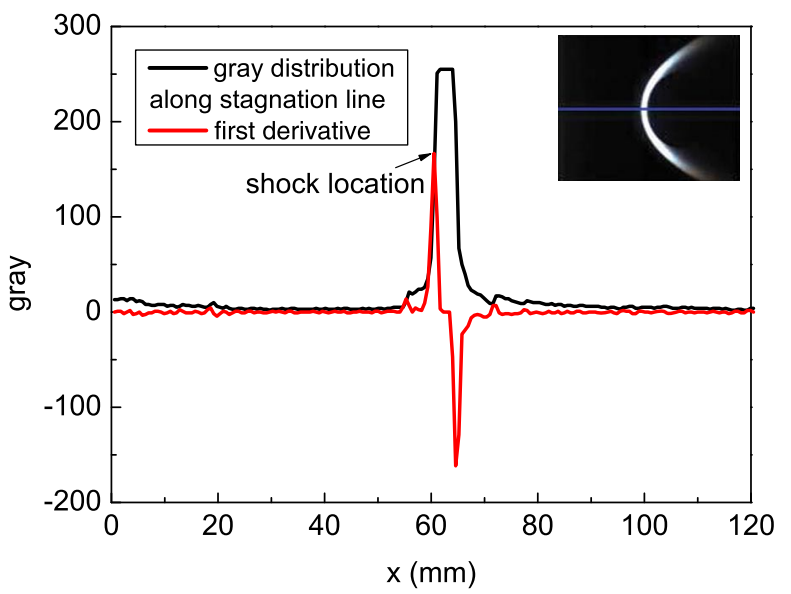

Fig. 3 Typical result of the imaging processing algorithm (inset: image of the shock produced by the semicylinder model). Blue line indicates row selected for processing.

model. To ensure that the model had not been displaced during the experiment, an image was also acquired just after the experiment. By comparing the processed results, we confirmed that no displacement of the model support system occurred during test time. This allowed the shock wave and model locations to be used for further analysis. The model fixing and model sting were sufficiently stiff so that no bending occurred during the run.

\section{Results and Discussions}

\section{A. Shock Shape}

\section{Semisphere}

Images of the flowfield evolution over the semisphere model are presented in Fig. 4a. The time interval between successive frames is $33 \mu \mathrm{s}$, and the exposure time is $1 \mu \mathrm{s}$. The flow in the image is from left to right. Detached shock waves are evident in the images. The image processing algorithm described previously was used to extract the shock and model locations: the results of which are shown in Fig. 4b. The normalized shock standoff distance $(\Delta / R)$ is derived from the shock and model locations. In Fig. $4 \mathrm{c}$, the shock shape is presented in the form of a normalized shock standoff distance as a function of the polar angle measured from the stagnation line. We found the following:

1) The shock standoff distance gradually decreases during the process in which the flowfield is established, and then it remains constant for a period of time before declining further. This means that the time taken to reach the quasi-steady state for this model is less than $33 \mu \mathrm{s}$, and the effective test time for this condition is less than $99 \mu \mathrm{s}$.
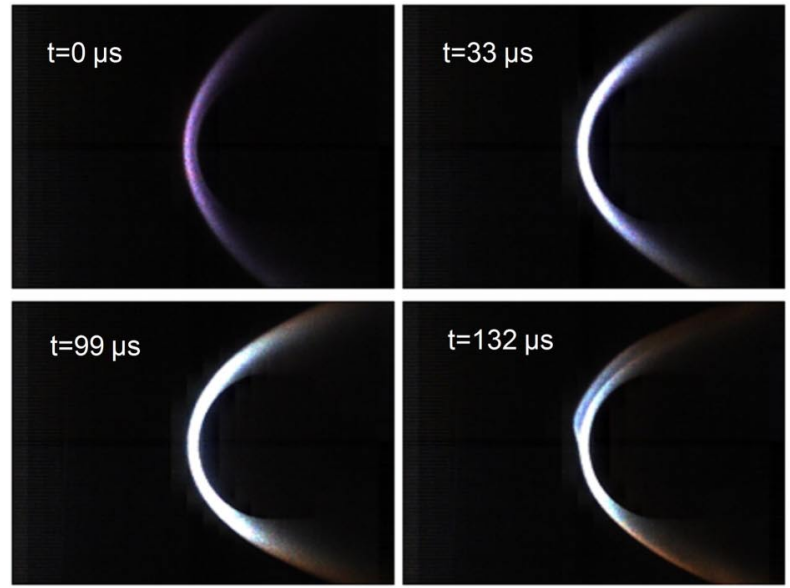

a)
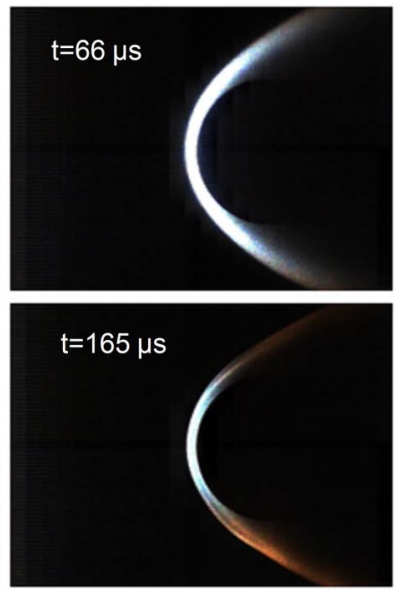
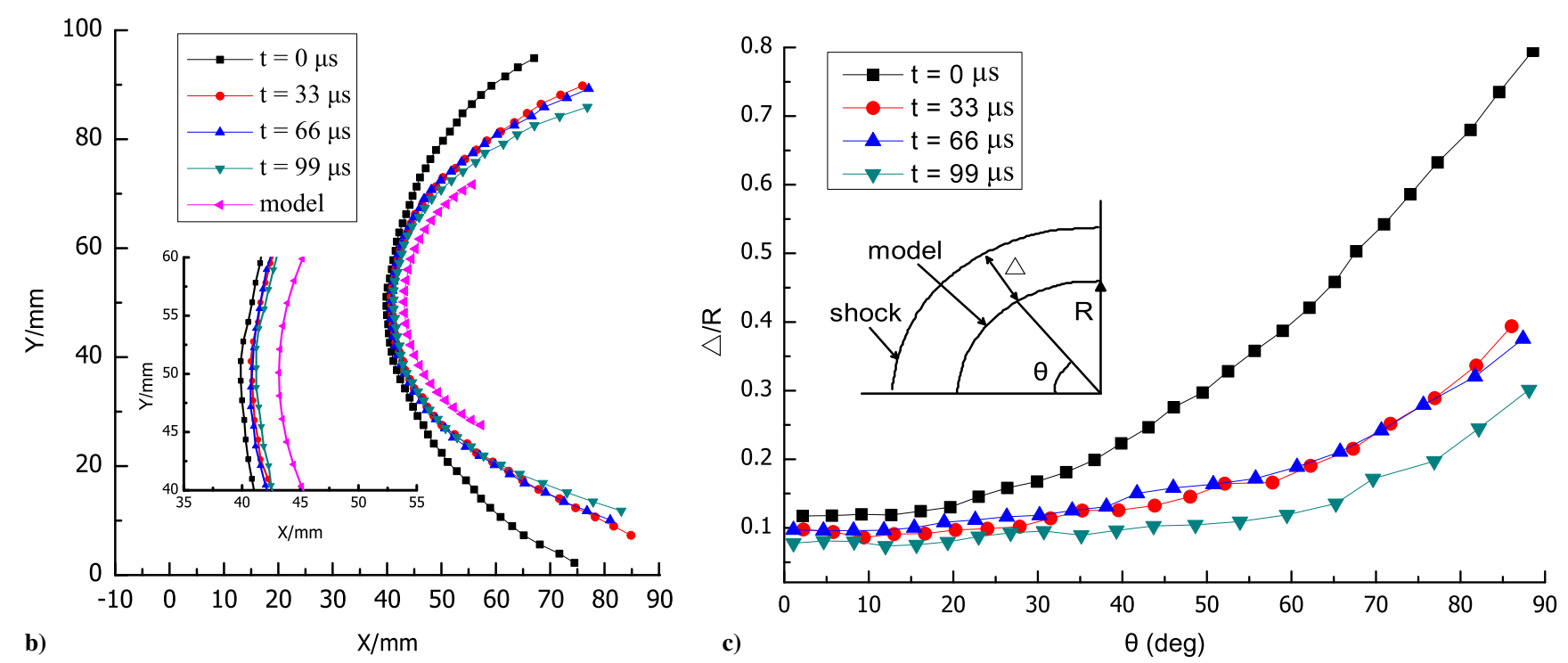

Fig. 4 Representations of a) evolution of visible shock layer over semisphere model, b) extracted shock and model locations, and c) normalized shock standoff distance versus polar angle as measured from stagnation line. 
2) During the effective test time, the normalized shock standoff distance along the stagnation line is $9.69 \%$. With increasing polar angle $\Theta$, the normalized shock standoff distance increases. When $\Theta$ equals $82 \mathrm{deg}$, the normalized shock standoff distance is about $33 \%$.

\section{Semicylinder}

The same experiment was performed with the semicylinder as the test model. The raw data of the evolution of the flowfield over the semicylinder model and the corresponding extracted shock and model locations can be found in Ref. [18]. The normalized standoff distance was also calculated and plotted in Fig. 5. The experimental results show the same trend as for Fig. 4. Quantitatively, the normalized shock standoff distance along the stagnation line is $17.96 \%$ during the effective test time. The shock standoff distance increases roughly 2.6 times when the polar angle is increased to $80 \mathrm{deg}$. The experimental results of both semisphere and semicylinder test models indicate that the shock shape is more sensitive to the flow states. Changes in the normalized shock standoff distance far from the stagnation point are more significant as compared with those near the stagnation point. Hence, it is more efficient and accurate to evaluate the CFD computation results using the whole shock shape.

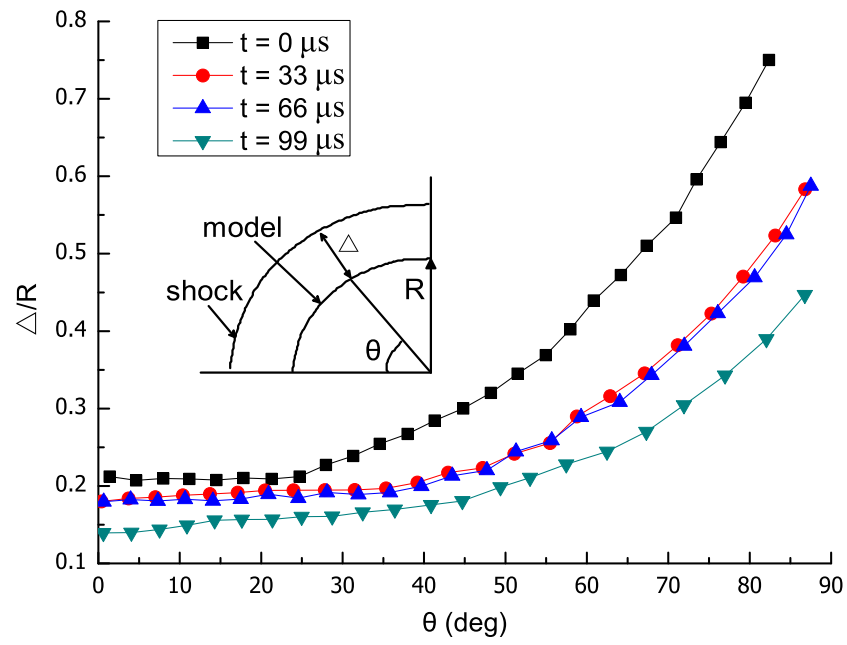

Fig. 5 Variation of normalized shock standoff distance of semicylinder with polar angle measured from stagnation line.
Table 2 Calculated shock angle

\begin{tabular}{lcc}
\hline \hline & \multicolumn{2}{c}{ Shock angle, deg. } \\
\cline { 2 - 3 } Time, $\mu$ s & Upper surface & Lower surface \\
\hline 0 & 62.2 & 70.2 \\
33 & 61.1 & 61.4 \\
66 & 62.1 & 61.0 \\
99 & 61.9 & 60.9 \\
\hline \hline
\end{tabular}

\section{Double Cone}

High-speed imaging data of the double-cone model are presented in Fig. 6a. The first cone is not easy to observe in the image because of the relatively low temperature in the shock layer. The bright area corresponds to the shock layer formed over the second cone. Shock and model locations are extracted in a similar manner to the semisphere model; see Fig. 6b. During the flow establishment process, the shock wave angle decreases. Based on the least-squares method, the data are further used to fit the wave angle. Table 2 shows the calculated results. From the $95 \%$ confidence bounds, the maximum uncertainty of the fitted angles is $\pm 1.2 \mathrm{deg}$. The distribution of the results is within the uncertainties of the fitting; therefore, it is difficult to estimate whether there is a shock oscillation.

The measured results of the semisphere and semicylinder show that the uniform flowfield is already terminated by the fourth image captured. However, the measured shock angle of the double cone features a slight variation. This indicates that, as compared with the semisphere and semicylinder models, the double-cone model is less sensitive to flow conditions. The intrinsic reason is the relatively low temperature of the shock layer. Therefore, under the same flow conditions, the calculation results from the blunt-body model are better in assessing the calculation accuracy of the CFD.

\section{Mars Science Laboratory Model}

A sequence of images of the flowfield over the simplified MSL model and the corresponding extracted shock locations is presented in Fig. 7. The results indicate clearly that the shock wave is relatively stable and the normalized shock standoff distance along the stagnation line is $9.59 \%$. Different from the experimental results of other models, the bright areas in the images taken by the high-speed camera increase with the test time.

\section{B. Shock-Shock Interaction}

Two double-wedge models, a 15-35 deg /45 deg double-wedge model and a 15-45 deg/55 deg double-wedge model, were
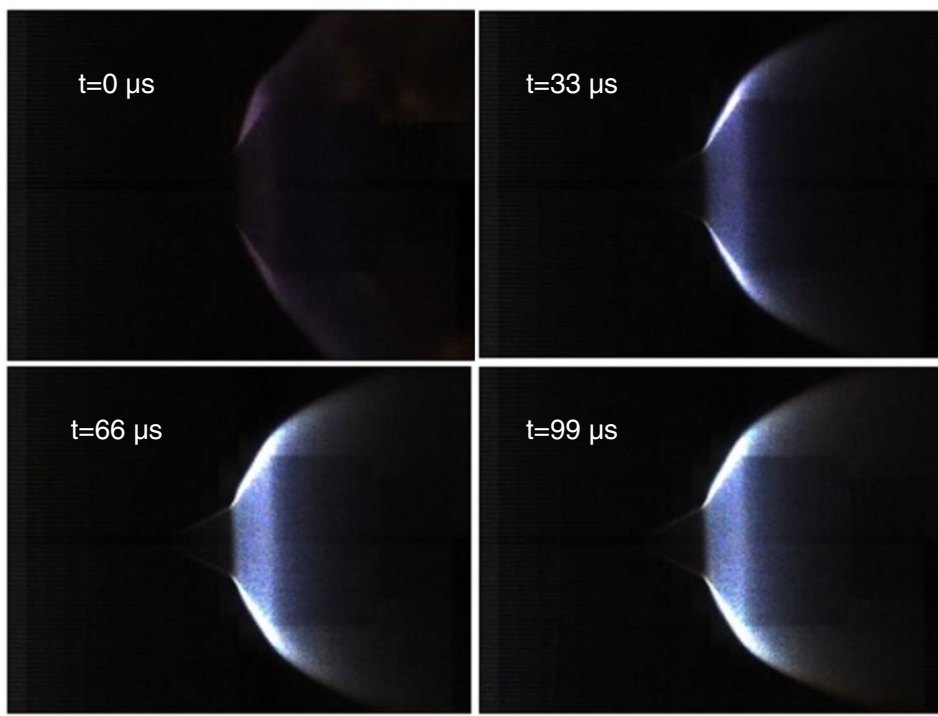

a)

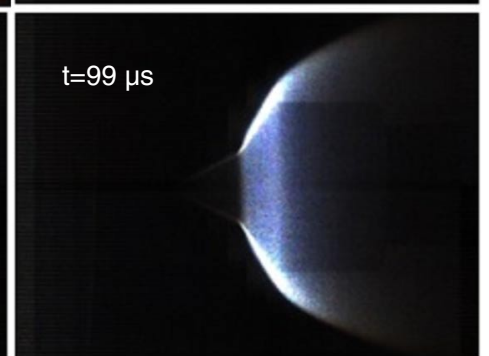

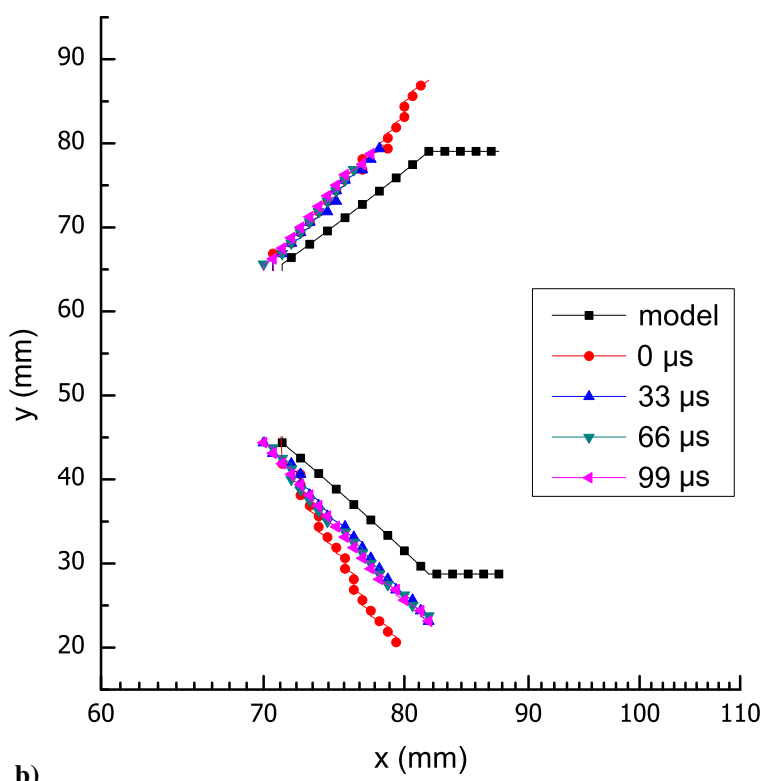

b)

Fig. 6 Representations of a) evolution of flowfield around double-cone model, and b) extracted shock and model locations. 

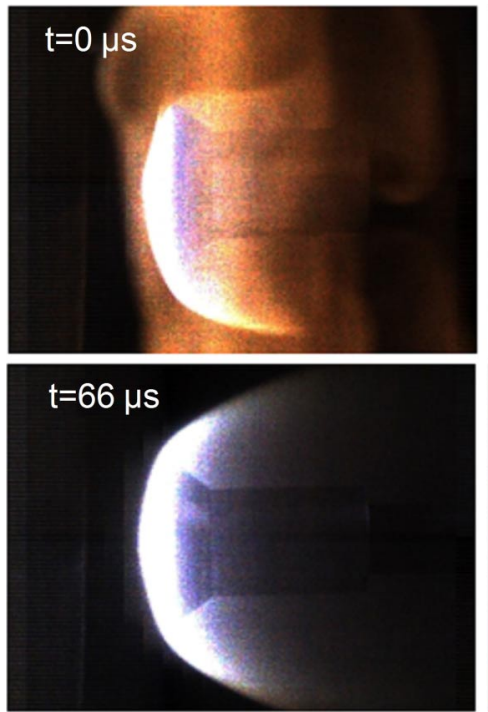

a)
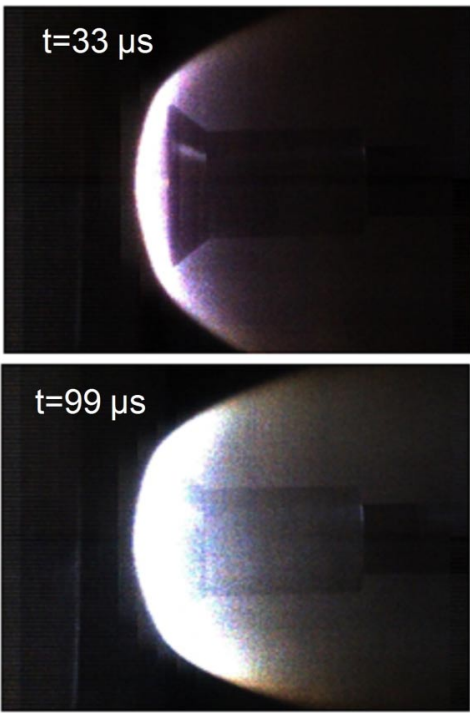

b)

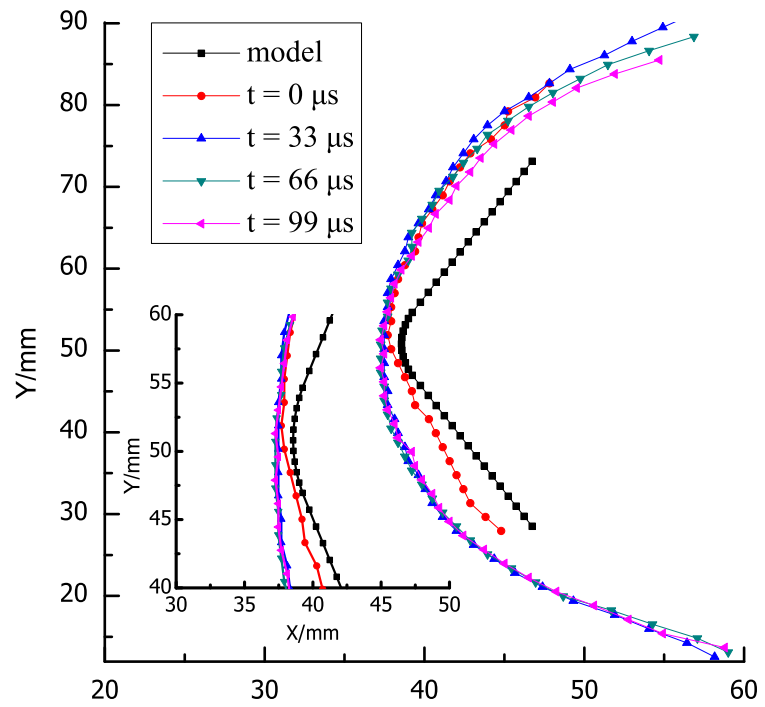

$\mathrm{X} / \mathrm{mm}$

Fig. 7 Representations of a) evolution of flowfield over simplified MSL model, and b) extracted shock and model locations.
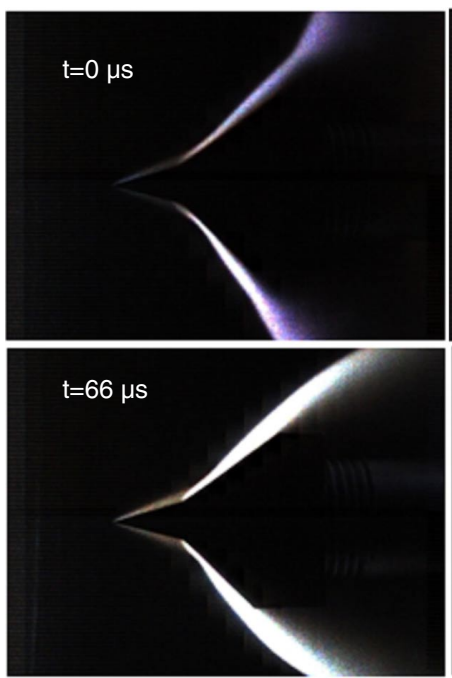

a)

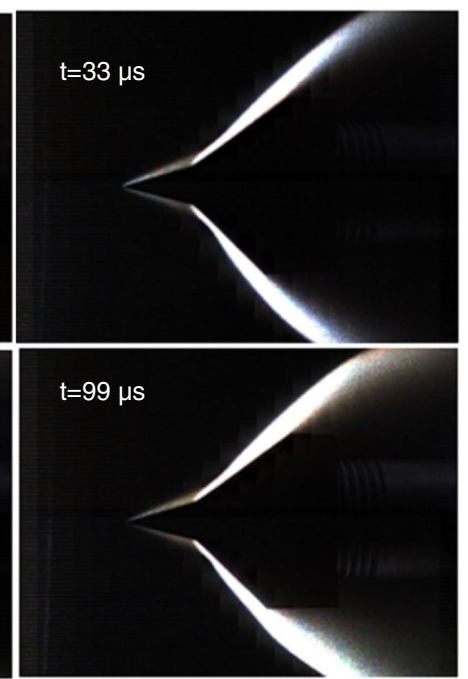

b)

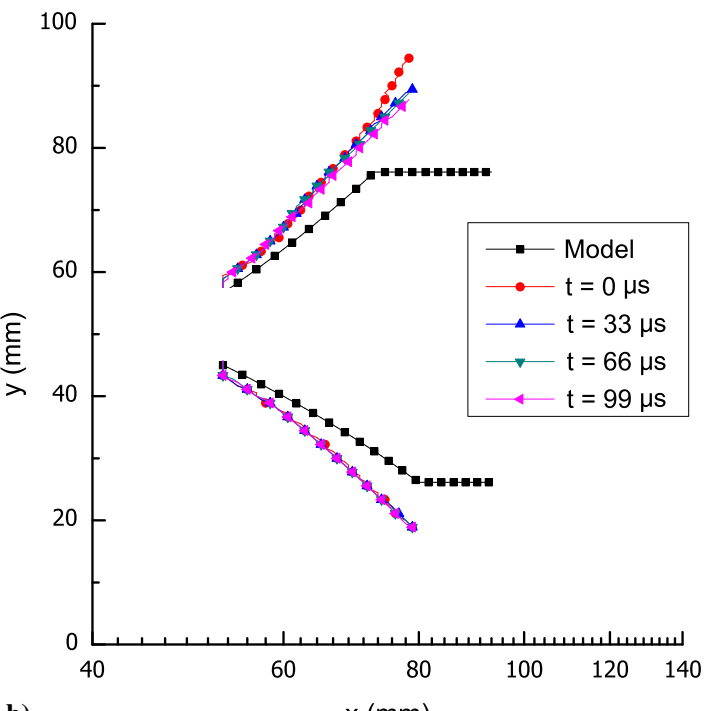

$x(\mathrm{~mm})$

Fig. 8 Representations of a) evolution of flowfield around a double wedge with second wedge angles of 35 and 45 deg, and b) extracted shock and model locations.

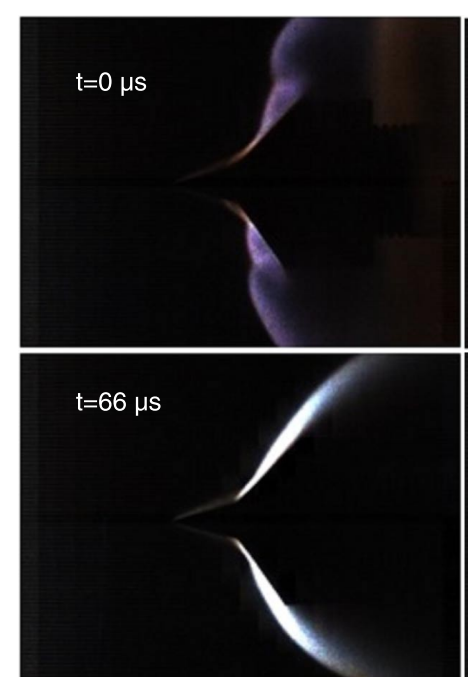

a)

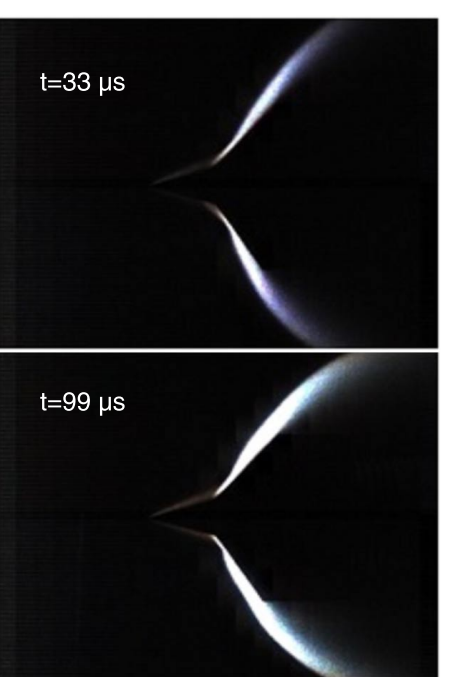

b)

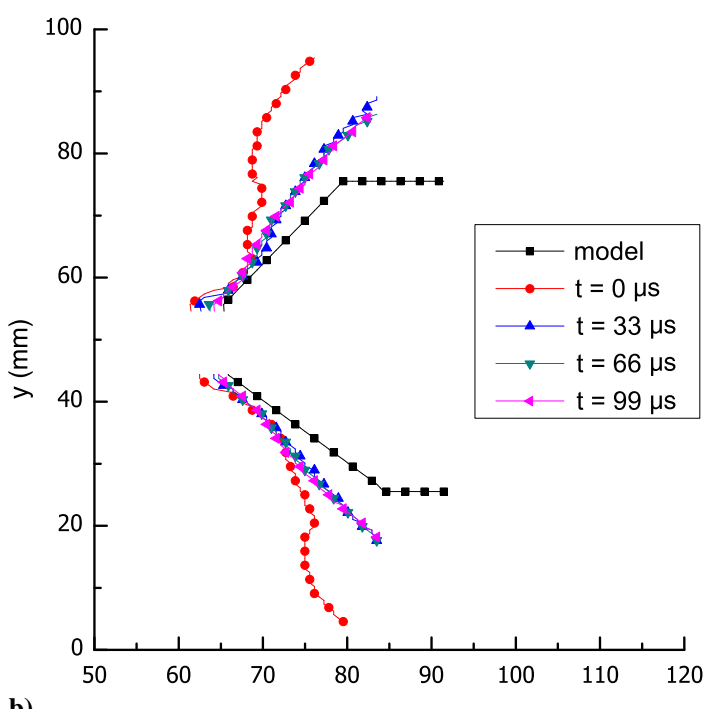

$x(\mathrm{~mm})$

Fig. 9 Representations of a) evolution of flowfield over double wedge with second wedge angles of 45 and 55 deg, and b) extracted shock and model locations. 


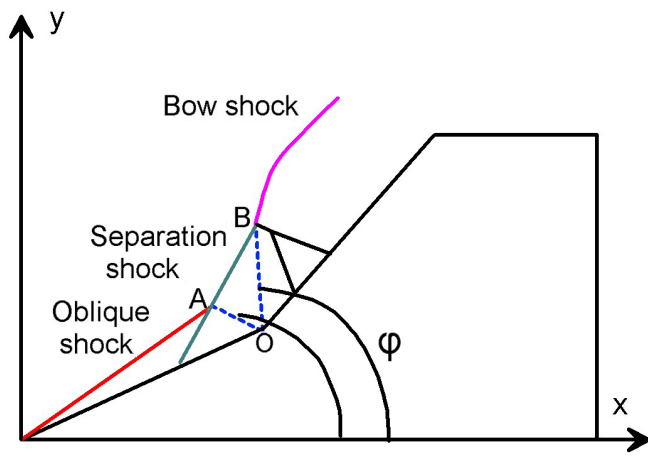

Fig. 10 Schematic of the flow over a double-wedge model and definitions of symbols used in the quantitative analysis.

Table 3 Extracted results of the shock-shock interaction

\begin{tabular}{lcccc}
\hline \hline Wedge angle, deg & $\varphi_{O A}, \operatorname{deg}$ & $\varphi_{O B}, \operatorname{deg}$ & $L_{O A}, \mathrm{~mm}$ & $L_{O B}, \mathrm{~mm}$ \\
\hline 35 & 69.4 & 48.4 & 4.8 & 13.4 \\
45 & 104.0 & 60.3 & 2.3 & 9.0 \\
55 & 135.0 & 69.4 & 1.6 & 4.9 \\
\hline \hline
\end{tabular}

selected to investigate the shock-shock interaction. Figures $\underline{8}$ and $\underline{9}$ present the experimental and quantitatively extracted results for these two models. Only the shock wave positions around the second wedge are given because the radiation from the first wedge is relatively weak. The flow over a double-wedge model (Fig. 10) is characterized by three shocks (oblique shock, separation shock, and bow shock), which can fortunately be identified in the experimental images. We therefore performed a quantitative analysis of the shock structure. The interaction points of the oblique shock and the separation shock and triple point, labeled as A and B in Fig. 10, were extracted. Due to the limited image resolution, the determination by eye seems to be the most reliable way to extract the positions of points A and B. The variations in the derived length $L$ and angle $\varphi$ of lines OA and OB with the second wedge angle are listed in Table 3. The influence of increasing the second wedge angle is summarized in the following:

1) Both $\varphi_{O A}$ and $\varphi_{O B}$ increase.

2) The value of the second wedge angle has a larger impact on $\varphi_{O A}$; increasing the second wedge angle from 35 to 55 deg increases $\varphi_{O A}$ and $\varphi_{O B}$ by 1.95 and 1.43 times, respectively.
Table 4 Chemical reactions considered

\begin{tabular}{lc}
\hline \hline Reaction no. & Reaction \\
\hline 1 & $\mathrm{~N}_{2}+\mathrm{M} \leftrightarrow 2 \mathrm{~N}+\mathrm{M}$ \\
2 & $\mathrm{O}_{2}+\mathrm{M} \leftrightarrow 2 \mathrm{O}+\mathrm{M}$ \\
3 & $\mathrm{NO}+\mathrm{M} \leftrightarrow \mathrm{N}+\mathrm{O}+\mathrm{M}$ \\
4 & $\mathrm{NO}+\mathrm{O} \leftrightarrow \mathrm{O}_{2}+\mathrm{N}$ \\
5 & $\mathrm{~N}_{2}+\mathrm{O} \leftrightarrow \mathrm{NO}+\mathrm{N}$ \\
6 & $\mathrm{~N}+\mathrm{O} \leftrightarrow \mathrm{NO}^{+}+\mathrm{e}^{-}$ \\
\hline \hline
\end{tabular}

3) The interaction points (A and B) both move upstream. Moreover, point $\mathrm{A}$ moves from downstream to upstream of hinge point $\mathrm{O}$ because $\varphi_{O A}$ is greater than $90 \mathrm{deg}$, whereas point $\mathrm{B}$ retains downstream.

4) Both $L_{O A}$ and $L_{O B}$ decrease, implying that the shock interaction point $A$ and triple point $B$ approach the model surface.

5) The shock wave around the second wedge changes from a straight oblique shock to a curved shock.

\section{Radiation Intensity Distribution}

The images taken at $66 \mu$ s were further analyzed to study the characteristics of the radiation intensity distribution. Figure 11 presents various intensity contours. Characteristic for all test models, the radiation contours display a self-similar structure. Moreover, the radiation intensity downstream of the shock layer decreases gradually.

Higher temperatures lead to stronger radiation. But, we do not think it is justifiable to explain the experimental results by this correlation. Therefore, CFD calculations were performed using the CFD ++ code from Metacomp Technologies, Inc., to guide the analysis of the experimental phenomenon. The calculation settings are as follows:

1) For the equation set, laminar compressible real-gas NavierStokes equations were used.

2) For the thermochemical model, seven species $\left(\mathrm{N}_{2}, \mathrm{O}_{2}, \mathrm{NO}, \mathrm{N}\right.$, $\mathrm{O}$, Nitrogen oxide ion $\left(\mathrm{NO}^{+}\right)$, and electron $\left(\mathrm{e}^{-}\right)$) were considered. The two-temperature model of Park [22] was used to determine the reaction rate coefficients. The chemical reactions considered are listed in Table 4.

3) For the boundary condition, no-slip and constant temperature (298 K) conditions were applied at the wall.

4) For the numerical algorithms, the backward Euler implicit method and the total variation diminishing method were used for time integration and spatial discretization, respectively.

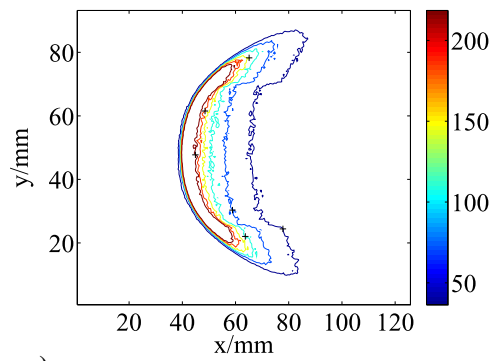

a)

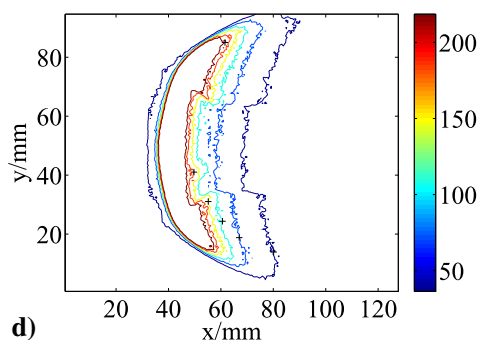

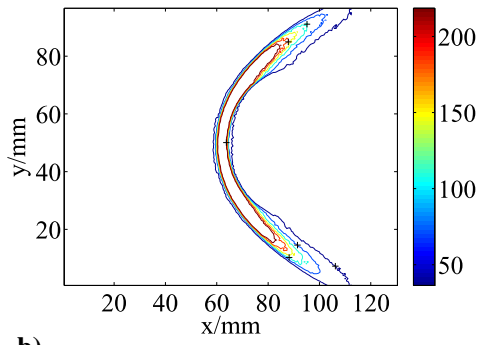

b)

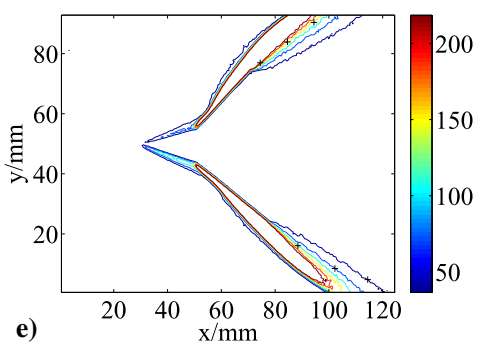

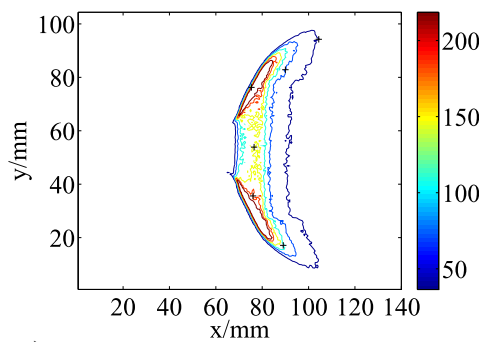

c)

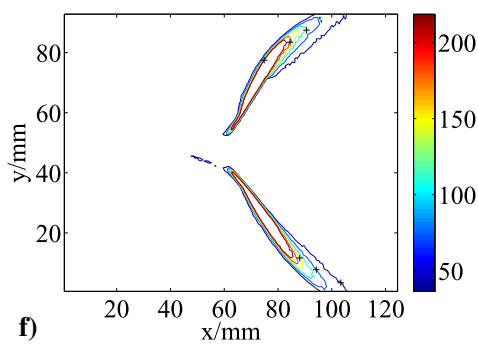

Fig. 11 Extracted radiation intensity contours from experimental images taken at $66 \mu \mathrm{s}:$ a) semisphere, b) semicylinder, c) double cone, d) simplified MSL model, e) double wedge with second wedge angles of 35 and $45 \mathrm{deg}$, and f) double wedge with second wedge angles of 45 and 55 deg. 

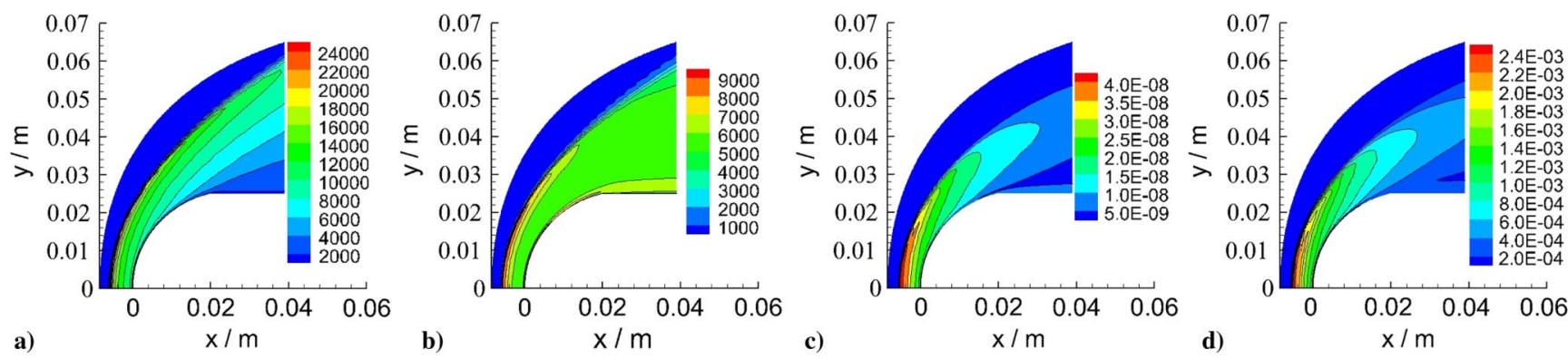

Fig. 12 CFD results of semisphere model: a) translational-rotational temperature, b) vibrational temperature, c) mass fraction of $e^{-}$, and d) mass fraction of $\mathrm{NO}^{+}$.

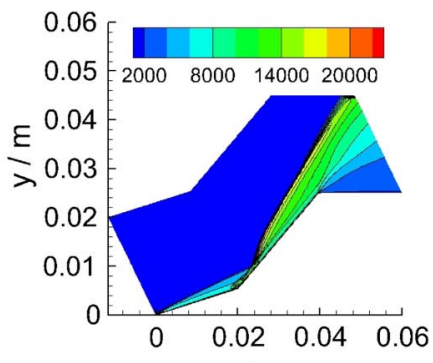

a)

$\mathrm{x} / \mathrm{m}$

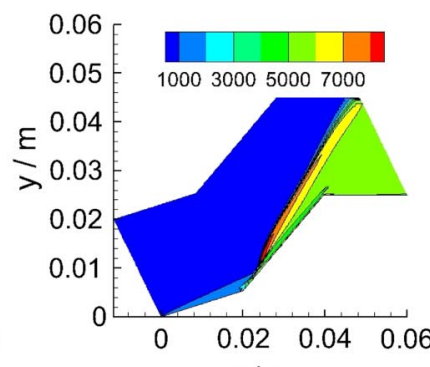

b)

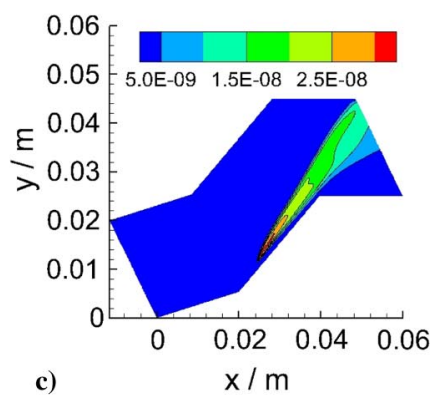

c)

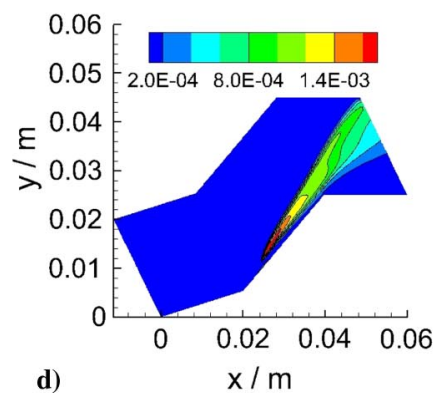

Fig. 13 CFD results of the 15-45 deg double-wedge model: a) translational-rotational temperature, b) vibrational temperature, c) mass fraction of $\mathrm{e}^{-}$, and d) mass fraction of $\mathrm{NO}^{+}$.

5) For the grid, from symmetry, only half of the geometry was modeled for the nominal angle of attack of zero design. The grid number was $151 \times 341$ for the $15-45$ deg double-wedge model and $201 \times 301$ for the semicylinder model. All the grids were constructed with a wall-normal spacing of $10 \mu \mathrm{m}$ at the body surface; the hyperbolic tangent function was selected to control grid point spacings in the normal direction.

The calculation results are in part presented in Figs. 12 and 13. The calculated shock standoff distance of the semicylinder is $5 . \overline{56} \mathrm{~mm}$, whereas the experimental result is $4.60 \mathrm{~mm}$. This deviation could be attributed to the radiation, which was not considered in the numerical simulation. Although the calculated shock structure is slightly different from the experiment results, we think it is helpful to explain radiation features qualitatively as a guide for the next step in the analysis. Compared with Figs. 11-13, the radiation intensity contours are very similar to those of $\mathrm{NO}^{+}$and $\mathrm{e}^{-}$. Hence, the radiation process is related to the chemical reaction of charged particles. Further studies are needed to reveal details of the radiation mechanism.

\section{Conclusions}

To extend the validation database of hypervelocity flow, experiments were performed in the JF-16 detonation-driven expansion tunnel. Details of the shock wave locations, shock standoff distance, shock-shock interaction characteristics, and radiation distributions were given. The experimental results also suggested the following:

1) The normalized shock shape downstream of the stagnation region is more sensitive to the flow condition than the normalized shock standoff distance at the stagnation point. Therefore, it is more efficient and accurate to evaluate the accuracy of the CFD results by comparing the whole shock shape.

2) For the same flow conditions, the numerical results of the bluntbody model can better assess the accuracy of the CFD simulation.

3) For the double-wedge model, increasing the second wedge angle moves the shock interaction point and the triple point upstream, and it draws the points nearer to the model surface.

4) The radiation intensity contours have a self-similar structure, and the radiation intensity downstream of the shock layer decreases gradually.

5) Preliminary simulation results showed that the radiation process was related to the chemical reactions of charged particles.

\section{Acknowledgment}

This work was supported by the National Natural Science Foundation of China (grant nos. 11602275, 11672312, 11672308, and 11532014).

\section{References}

[1] Gnoffo, P. A., Weilmuenster, K. J., Hamilton, H. H., Olynick, D. R., and Venkatapathy, E., "Computational Aerothermodynamic Design Issues for Hypersonic Vehicles," Journal of Spacecraft and Rockets, Vol. 36, No. 1, 1999, pp. 21-43. https://doi.org/10.2514/2.3430

[2] Eichmann, T., Brandis, A., Potter, D., McIntyre, T., and RubinszteinDunlop, H., "Radiating Hypersonic Flow Studies Using a Super-Orbital Expansion Tube," 26th AIAA Aerodynamic Measurement Technology and Ground Testing Conference, AIAA Paper 2008-4135, 2008. https://doi.org/10.2514/6.2008-4135

[3] Grinstead, J. H., Wilder, M. C., Reda, D. C., Cruden, B. A., and Bogdanoff, D. W., "Advanced Spectroscopic and Thermal Imaging Instrumentation for Shock Tube and Ballistic Range Facilities," NASA Ames Research Center RTO-EN-AVT-186, ARC-E-DAATN1396, Moffett Field CA, 2010, https://ntrs.nasa.gov/search.jsp? $\mathrm{R}=20100027326$ [retrieved 13 Jan. 2020].

[4] Nonaka, S., Mizuno, H., Takayama, K., and Park, C., "Measurement of Shock Standoff Distance for Sphere in Ballistic Range," Journal of Thermophysics and Heat Transfer, Vol. 14, No. 2, 2000, pp. 225-229.

https://doi.org/10.2514/2.6512

[5] Belouaggadia, N., Hashimoto, T., Nonaka, S., Takayama, K., and Brun, R., "Shock Detachment Distance on Blunt Bodies in Nonequilibrium Flow," AIAA Journal, Vol. 45, No. 6, 2007, pp. 1424-1429. https://doi.org/10.2514/1.17806

[6] Olivier, H., "A Theoretical Model for the Shock Stand-Off Distance in Frozen and Equilibrium Flows," Journal of Fluid Mechanics, Vol. 413, June 2000, p. 345-353. https://doi.org/10.1017/S0022112000008703

[7] Wen, C.-Y., and Hornung, H. G., "Non-Equilibrium Dissociating Flow over Spheres," Journal of Fluid Mechanics, Vol. 299, Sept. 1995, pp. 389-405. https://doi.org/10.1017/S0022112095003545

[8] Sinclair, J., and Cui, X., "A Theoretical Approximation of the Shock Standoff Distance for Supersonic Flows Around a Circular Cylinder," Physics of Fluids, Vol. 29, No. 2, 2017, Paper 026102. https://doi.org/10.1063/1.4975983 
[9] Zander, F., Gollan, R. J., Jacobs, P. A., and Morgan, R. G., "Hypervelocity Shock Standoff on Spheres in Air," Shock Waves, Vol. 24, No. 2, 2014, pp. 171-178. https://doi.org/10.1007/s00193-013-0488-x

[10] Holden, M., Wadhams, T., Smolinski, G., MacLean, M., Harvey, J., and Walker, B., "Experimental and Numerical Studies on Hypersonic Vehicle Performance in LENS Shock and Expansion Tunnels," 44th AIAA Aerospace Sciences Meeting and Exhibit, AIAA Paper 20060125, 2006.

https://doi.org/10.2514/6.2006-125

[11] Holden, M., Wadhams, T., MacLean, M., Dufrene, A., Mundy, E., and Marineau, E., "Review of Basic Research and Development Programs Conducted in the LENS Facilities in Hypersonic Flows," 50th AIAA Aerospace Sciences Meeting Including the New Horizons Forum and Aerospace Exposition, AIAA Paper 2012-0469, 2012. https://doi.org/10.2514/6.2012-469

[12] Knight, D., Longo, J., Drikakis, D., Gaitonde, D., Lani, A., Nompelis, I., Reimann, B., and Walpot, L., "Assessment of CFD Capability for Prediction of Hypersonic Shock Interactions," Progress in Aerospace Sciences, Vols. 48-49, Jan. 2012, pp. 8-26. https://doi.org/10.1016/j.paerosci.2011.10.001

[13] Olejniczak, J., Candler, G., Wright, M., Hornung, H., and Leyva, I., "High Enthalpy Double-Wedge Experiments," Advanced Measurement and Ground Testing Conference, AIAA Paper 1996-2238, 1996. https://doi.org/10.2514/6.1996-2238

[14] Park, C., "Validation of CFD Codes for Real-Gas Regime," 32nd Thermophysics Conference, AIAA Paper 1997-2530, 1997. https://doi.org/10.2514/6.1997-2530

[15] Hao, J., Wang, J., and Lee, C., "Numerical Simulation of High-Enthalpy Double-Cone Flows," AIAA Journal, Vol. 55, No. 7, 2017, pp. 24712475.

https://doi.org/10.2514/1.J055746
[16] Karl, S., Schramm, J. M., and Hannemann, K., "High Enthalpy Cylinder Flow in HEG: A Basis for CFD Validation," 33rd AIAA Fluid Dynamics Conference and Exhibit, AIAA Paper 2003-4252, 2003. https://doi.org/10.2514/6.2003-4252

[17] Jiang, Z., Wu, B., Gao, Y., Zhao, W., and Hu, Z., "Development of the Detonation-Driven Expansion Tube for Orbital Speed Experiments," Science China Technological Sciences, Vol. 58, No. 4, 2015, pp. 695700. https://doi.org/10.1007/s11431-014-5756-1

[18] Yuan, C. K., Zhou, K., Liu, Y. F., Hu, Z. M., and Jiang, Z. L., "Spectral Measurements of Hypervelocity Flow in an Expansion Tunnel," Acta Mechanica Sinica, Vol. 35, No. 1, 2019, pp. 24-31. https://doi.org/10.1007/s10409-018-0806-8

[19] Hu, Z., Wang, C., Jiang, Z., and Khoo, B., "On the Numerical Technique for the Simulation of Hypervelocity Test Flows," Computers and Fluids, Vol. 106, Jan. 2015, pp. 12-18. https://doi.org/10.1016/j.compfluid.2014.09.039

[20] Sasoh, A., Ohnishi, Y., Ramjaun, D., Takayama, K., Otsu, H., and Abe, T., "Effective Test Time Evaluation in High-Enthalpy Expansion Tube," AIAA Journal, Vol. 39, No. 11, 2001, pp. 2141-2147. https://doi.org/10.2514/2.1210

[21] Swantek, A. B., and Austin, J. M., "Flowfield Establishment in Hypervelocity Shock-Wave/Boundary-Layer Interactions," AIAA Journal, Vol. 53, No. 2, 2015, pp. 311-320. https://doi.org/10.2514/1.J053104

[22] Park, C., "Assessment of Two-Temperature Kinetic Model for Ionizing Air," Journal of Thermophysics and Heat Transfer, Vol. 3, No. 3, 1989 , pp. 233-244.

https://doi.org/10.2514/3.28771

C. Wen Associate Editor 